\title{
Business and Social Behaviour Intelligence Analysis Using PSO
}

\author{
Vinay S Bhaskar ${ }^{1}$, Abhishek Kumar Singh ${ }^{2}$, Jyoti Dhruw ${ }^{3}$, Anubha Parashar ${ }^{4}$, Mradula Sharma ${ }^{5}$ \\ ${ }^{1}$ Human Resource Manager, Electro Equipment, Roorkee, India \\ ${ }^{2}$ IIIT Allahabad \\ ${ }^{3}$ Chhatrapati Shivaji Institute of Technology (CSIT, Durg, Chhattisgrah \\ ${ }^{4}$ Maharshi Dayanand University, Rohtak \\ ${ }^{5}$ MNNIT Alllahabad
}

\begin{abstract}
The goal of this paper is to elaborate swarm intelligence for business intelligence decision making and the business rules management improvement. The paper introduces the decision making model which is based on the application of Artificial Neural Networks (ANNs) and Particle Swarm Optimization (PSO) algorithm. Essentially the business spatial data illustrate the group behaviors. The swarm optimization, which is highly influenced by the behavior of creature, performs in group. The Spatial data is defined as data that is represented by 2D or 3D images. SQL Server supports only 2D images till now. As we know that location is an essential part of any organizational data as well as business data: enterprises maintain customer address lists, own property, ship goods from and to warehouses, manage transport flows among their workforce, and perform many other activities. By means to say a lot of spatial data is used and processed by enterprises, organizations and other bodies in order to make the things more visible and selfdescriptive. From the experiments, we found that PSO is can facilitate the intelligence in social and business behaviour.
\end{abstract}

Keywords - PSO, Map, Artificial Intelligence, Geography, Optimization.

\section{INTRODUCTION}

WARM describes a behavior of an aggregate of animals of $\checkmark$ similar size and body orientation [1]. Swarm intelligence (SI) is based on the collective behavior of a group of animals. Collective intelligence emerges via grouping and communication, resulting in successful foraging (the act of searching for food and provisions) for individual in the group, for examples Bees, ants, termites, fishes, birds etc. The perform the following sequence of activity in group: Marching of ants in an army, Birds flocking in high skies, Fish school in deep waters, Foraging activity of micro-organisms.In the context of AI, SI systems are based on collective behavior of decentralized, self-organized systems [2]. Typically made up of a population of simple le agents interacting with one another locally and with their environment causing coherent functional global pattern to emerge. Distributed problem solving model without centralized control. Even with no centralized control structure dictating how individual agents should behave, local interactions between agents lead to the emergence of complex global behavior [3]. Swarms are powerful which can achieve things which no single individual could do.

An intelligent technology is the duplication of human thought process by machine. It learn from experience, interpreting ambiguities, rapid response to varying situations, applying reasoning to problem-solving and manipulating by applying knowledge, thinking and reasoning [4]. Different from traditional optimization technique, evolutionary computation techniques work on a population of potential solutions (points) of the search space. The most commonly used population-based evolutionary computation techniques is PSO [5]. It is a cost optimized solution. Organizations generate and collect large volumes of data, which they use in daily operations. Yet despite this wealth of data, many organizations have been unable to fully capitalize on its value because information implicit in the data is not easy to distinguish. However, to compete effectively today, taking advantage of high-return opportunities in a timely fashion, decision-makers must be able to identify and utilize the information. These requirements imply that an intelligent system must interact with a data warehouse and must interface with decision support systems (DSS), which are used by decision-makers in their daily activities. There is a substantial amount of empirical evidence that human intuitive judgment and decision-making can be far from optimal, and it deteriorates even further with complexity and stress. Because in many situations the quality of decisions is important, aiding the deficiencies of human judgment and decision-making has been a major focus of science throughout history [6] [7]. Disciplines such as statistics, economics, and operations research developed various methods for making rational choices. More recently, these methods, often enhanced by a variety of techniques originating from information science, cognitive psychology, and artificial intelligence, has been implemented in the form of computer programs as integrated computing environments for complex decision making. Such environments are often given the common name of decision support systems (DSS) [20] [21].

The development and deployment of managerial decision support system represents an emerging trend in the business and organizational field in which the increased application of Decision Support Systems (DSS) can be compiling by 
Intelligent Systems (IS). Decision Support Systems (DSS) are a specific class of computerized information system that supports business and organizational decision-making activities [18] [19]. A properly designed DSS is an interactive software-based system intended to help decision makers compile useful information from raw data, documents, personal knowledge, and/or business models to identify and solve problems and make decisions. Competitive business pressures and a desire to leverage existing information technology investments have led many firms to explore the benefits of intelligent data management solutions such as Particle Swarm Optimization (PSO). This study proposes a new PSO (SPSO)-model based on product mix model for optimizing Constraint values as well as objective function. The formulations of the objective function for the minimization problem. This technology is designed to help businesses to finding multi objective functions, which can help to understand the purchasing behavior of their key customers, detect likely credit card or insurance claim fraud, predict probable changes in financial markets, etc. Keywords: Linear problem, Intelligent System, particle swarm optimization, simplex method. The Sql Server Spatial Database is designed to make the storage, retrieval, analysis and manipulation of spatial data easier and natural to users. Once we have data we can perform any operation easily like retrieve all data related to our concern, manipulate it as par requirement [17]. Spatial data is the main need for graphic visualization to make useful result about. It is useful to guess of localization i.e. longitude and latitude. Now we have Microsoft SQL Server 2008 which is including simple feature graphical representation of location data as location in the map by firing query for location in query results area which was not available in the previous version of Microsoft SQL Server i.e. Microsoft SQL Server 2000 and 2005. This visualizer works with a geography column in the query results and in graph by plotting location data and if multiple location column in appearing in as a query result we can select one to visualize [22] [23] [24]. For example suppose we have a location data then we have a choice to flexible plot the diagram of the map either it is rectangle or any other polygon shape for projection purpose on graph but we are assuming out projection should not be overlaid with default projection. By this technique we can easily and very convenient overlay a graph on map if we have the table of map outline data which we can use to do UNION ALL between the row set and the row set that have the map location [8]. Spatial data is useful in lots of cases because every customer has its own address. We usually think of addresses as street, city, state, country and ZIP code and in other words spatial data can use for finding the exact position of real world entity like suppose we have the database of customer where we are storing address which not merely containing which we are supposing street, city or state but actually it is showing the latitude and longitude. As technically by address we mean part of territory which can draw by polygon for convenient we are assuming single point not a polygon and by this we can guess about longitude and latitude for the answer of query like here:

- Find the nearest branch of bank for client info.
- And who is the representative or concern person for that particular client.

- And we can also retrieve the client information within a particular boundary i.e. how many clients are there in particular organisation of any business.

We can guess client information related to position within the range and outside so it is not just maintain the branch location of our office but also allowing to put information of client related to us. To make whole system and process convenient, reliable, to speedup of process, robust by storing data and analyzed data and get information and by exchanging information we can assure all the above mention benefit in our work. As we know that reliability, speed and robustness is only the required feature; here we will consider. Another important aspect or we can say the further advancement of spatial techniques is the visualization of spatial data using maps. The methods used for retrieval problem [9] [10], human activity and face recognition problem [11] [12], location estimation, [13] and scalable replica estimation problem [14] can also be integrated with proposed PSO based approach. After all, location information is all about maps, and to paraphrase a common saying, a map is certainly worth 1,000 words. A better choice is to use a visualizer that provides map overlay by default [15] [16]. SQL Server Studio 2008 use a map overlay for showing spatial data. Some other approaches are also used for similar task [25] [26] [27] [28]. The Advantages of proposed method are as follows: (1) Adaptability - Selforganizing, (2) Robustness - Ability to find a new solution if the current solution becomes invalid, (3) Reliability - Agents can be added or removed without disturbing behavior of the total system because of the distributed nature, (4) Simplicity, and (5) No central control. The rest of the paper is organized in further four section: section 2 discuss some background concepts which are used in our problem such as spatial data, visulizers and spatial index; section 3 introduced the proposed methodology of business intelligence using the concept of particle swarm optimization; section 4 shows some practical application of introduced work with result; finally section 5 conclude the paper with future remark of the paper.

\section{BACKGROUND CONCEPTS}

\section{A. Spatial data}

Spatial data is data which is use for finding the position of the real world entity like we have to find the position of sea sore, restaurant, hotel, tourist palace, historical important location and some territory. In spatial database is the combination of all the data types, statics and indexing of location. For fast accessing, the location information from spatial database done by spatial function and spatial indexing. We can retrieve it through $\mathrm{Sql}$. 
DECLARE @addr nvarchar(256) = 'Some sample address, City, State, Zip';

DECLARE @addr_as_xy nvarchar(30);

DECLARE @g geography;

SET @addr_as_xy=dbo.Geocoder(@addr);

SET @g= geography::STPointFromText(@addr_as_xy, 4326);

Fig. 1. Code for location initialization

We have an instance of a location type with a sql variable declaration (@p position) or a column of table, and we have a number of way to initialize data type let's us take an example we have geographical data type who is using to show the instance of location point, the easier way to do it by use of STPointfromText() method of the location type. The STPointFromText() method is using for SRID (spatial reference identifier) with other feature textual representation of point that is POINT(x,y) in open Geospatial boundary. SRID identifier is the spatial reference system for use of either shape of earth whether flat or round mapping and it is enough to know the MapPoint geocoder Web Service uses coordinates of GPS to related to SRID 4326 so for location initialization our code will look something like in Figure 1:

\section{B. Visualizers}

Microsoft SQL Server 2008 provides a feature to visualize location result in either query result area or window. Microsoft SQL Server 2008 shows the spatial data as a graph plot in query result area to represent the longitude and latitude related to geography and we can select one column if we have lot of spatial column appearing in same time by query result. And column to display should be in SQL Server binary format, the ToString() method or STAsText() method not work with visualizer.

\section{Spatial Indexes}

After growing our enterprise to calculate the position between client and every entrepreneur and every salesman and every client might be too slow. Microsoft SQL Server 2008 has also spatial indexing. And here spatial indexing is based on ordinary B-tree index to make it faster like as relational indexes in SQL Server 2000 and 2005.If we have geographical data type, then we are dividing the entire globe into hemisphere and projecting each hemisphere onto a plane. And if we have geometrical data type, because we are specifying our own rectangular coordinate system, we can specify the boundaries that our spatial index covers the whole area. To return to the customer system, you could define a spatial index on the geog column in your customer table with the following data definition language (DDL):

CREATE SPATIAL INDEX cust_geog_idx

ON dbo.customer(geog)
GRIDS

$=($ LEVEL1 $=$ HIGH, LEVEL2 $=$ HIGH, LEVEL3 $=$ HIGH, LEVEL $4=\mathrm{HIGH})$ ).

\section{MethODOLOGY}

\section{A. Swarm inspired methods}

Particle Swarm Optimization (PSO): PSO is a population based stochastic optimization technique developed by Eberhart and Kennedy in 1995. It is inspired by social behaviour of flocks of birds and school of fish. It is a set of agents (similar to ants), search in parallel for good solutions and co-operate through the pheromone-mediated indirect method of communication. They belong to a class of meta-heuristics. These systems started with their use in the Travelling Salesman Problem (TSP). They have applications to practical problems faced in business and industrial environments. The evolution of computational paradigm for an ant colony intelligent system (ACIS) is being used as an intelligent tool to help researchers solve many problems in areas of science and technology.

Particle Swarm Intelligent Systems: Originated with the idea to simulate the unpredictable choreography of a bird flock with Nearest-neighbour velocity matching, Multi-dimensional search, Acceleration by distance, and Elimination of ancillary variables. PSO shares many similarities with Genetic Algorithms (GA). The system is initialized with a population of random solutions (called particles) and searches for optima by updating generations. Each particle is assigned a randomized velocity. Particles fly around in a multidimensional search space or problem space by following the current optimum particles. However, unlike GA, PSO has no evolution operators such as crossover and mutation. Compared to GA, the advantages of PSO are that it is easy to implement and there are few parameters to adjust. Each particle adjusts its position according to its own experience and the experience of a neighboring particle. Particle keeps track of its co-ordinates in the problem space which are associated with the best solution/ fitness achieved so far along with the fitness value (pbest $\leftarrow$ partcle best). Overall best value obtained so far is also tracked by the global version of the particle optimizer along with its location (gbest). There are two versions exist (according to acceleration): (1) Global - At each time step, the particle changes its velocity (accelerates) and moves towards its pbest and gbest and (2) Local - In addition to pbest, each particle also keeps track of the best solution (lbest/nbest - neighbour best) attained within a local topological neighbourhood of the particle. The acceleration thus depends on pbest, lbest, and gbest.

\section{B. Problem solution}

Conceptual framework of sense making (Psychological Systems): A psychological system can be thought of as an "information-processing" function. We measure psychological systems by identifying points in psychological space. Usually the psychological space is considered to be multidimensional. 
A swarm is a large number of homogenous, simple agents interacting locally among themselves, and their environment, with no central control to allow a global interesting behavior to emerge. Swarm-based algorithms have recently emerged as a family of nature-inspired, population-based algorithms that are capable of producing low cost, fast, and robust solutions to several complex problems. This indirect type of interaction is referred to as stigmergy, which essentially means communication through the environment.

"Philosophical Leaps" Required:

i. Individual minds = a point in space

ii. Multiple individuals can be plotted in a set of coordinates

iii. Measuring the individuals result in a "population of points"

iv. Individuals near each other imply that they are similar

v. Some areas of space are better than others Location.

Applying Social Psychology: Individuals (points) tend to move towards each other and influence each other. This is why; individuals want to be in agreement with their neighbors. Individuals (points) are influenced by their previous actions/behaviors and the success achieved by their neighbors. Figure 2 illustrates the working algorithm of particle swarm optimization. First the particle is initialized randomly. Then for each particle a fitness value is calculated. If the current fitness value is better than previous personal best then personnel based is set to current fitness value. The global best is also updated with the best fitness value. After updating the personnel best and global best, particle velocity is calculated using equation (a) of Figure 3 and [particle position is also updated using equation (b) of Figure 3. These steps are repeated until the convergence criteria is met (i.e. optimized solution or maximum iteration).

\section{PRACTICAL WORK AND RESULTS}

Human being is a social animal and one of intelligent creation of god. The Human being whose behavior is strongly inspired by and govern my group activity like some animal fish schooling, bird flocking swam optimization. Whose behavior is influence by group.As there is a population which has their own knowledge of intelligence and best fit of cost of their daily need but if some trend can show them a different need and arise some tread which can motivate or can given some general scene about our prediction. Particle Swarm is an evolutionary computation based technique. There is a substantial amount of empirical evidence that human intuitive judgment and decision-making can be far from optimal, and it deteriorates even further with complexity and stress. Because in many situations the quality of decisions is important, aiding the deficiencies of human judgment and decision-making has been a major focus of science throughout history. Disciplines such as statistics, economics, and operations research developed various methods for making rational choices. More recently, these methods, often enhanced by a variety of techniques originating from information science, cognitive psychology, and artificial intelligence, has been implemented in the form of computer programs as integrated computing environments for complex decision making. Such environments are often given the common name of decision support systems (DSS).

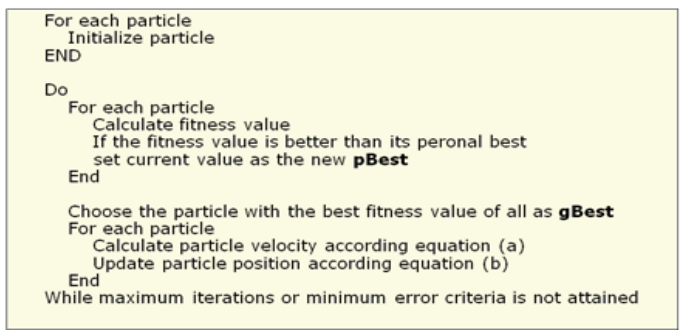

Fig. 2. Working algorithm of PSO

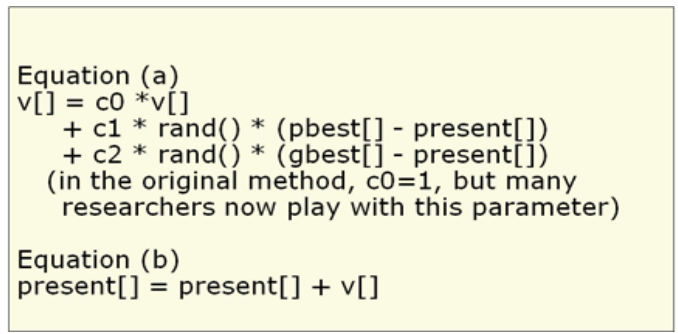

Fig. 3. Velocity updating of a swarm in PSO

A PSO based algorithm is developed to define the bi-level pricing model. Experiments illustrate that thus PSO based algorithm can achieve a profit increase for buyers or vendors, if they are treated as leader under some situation (see Figure 4).

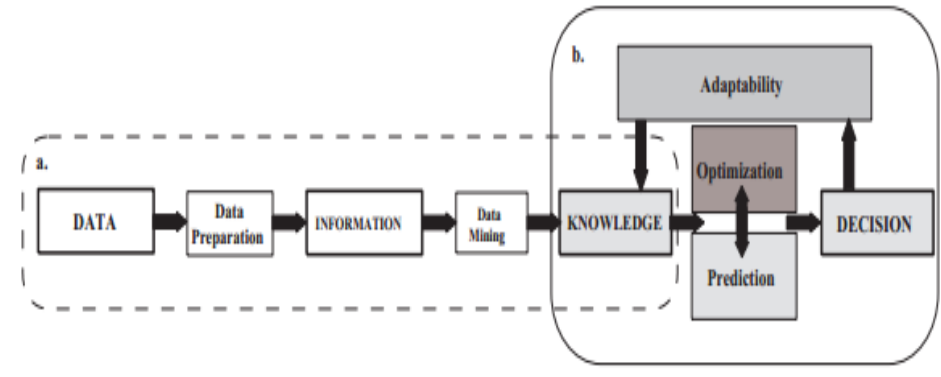

Fig. 4. PSO based Algorithm

Figure 5 shows points representing a set of more than 700 cities from the Mondial database in the SQL Server Management Studio visualizer.

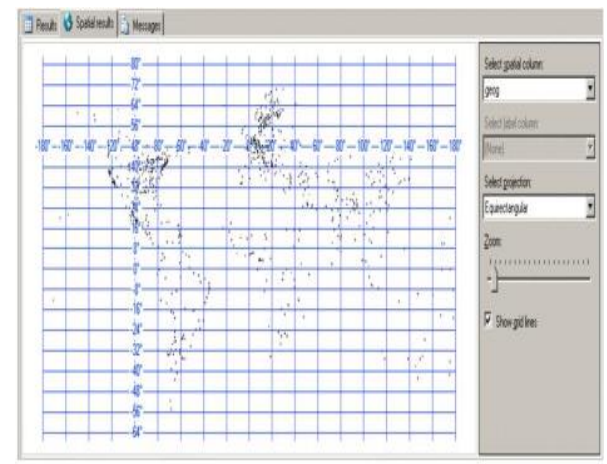

Fig. 5. Output of SQL Server query (A) 
Following are the two SQL server queries and its output.

The SQL Server query (A): (see output in Figure 5)

SELECT geog, name

FROM Mondial.dbo.city

WHERE geog IS NOT NULL

The SQL Server query (B): (see output in Figure 6)

SELECT geo, name

FROM Mondial.dbo.cityname

WHERE geo IS NOT NULL

UNION ALL

SELECT geo, cntry_name

FROM SpatialSamples.dbo.cntry

An even better choice is to use a commercial or shareware visualizer that provides map overlay by default, as shown in Figure 5. We see that the Spatial Results tab in MS SQL Server 2008 shows a rowset of more than 600 points with a map overlay.

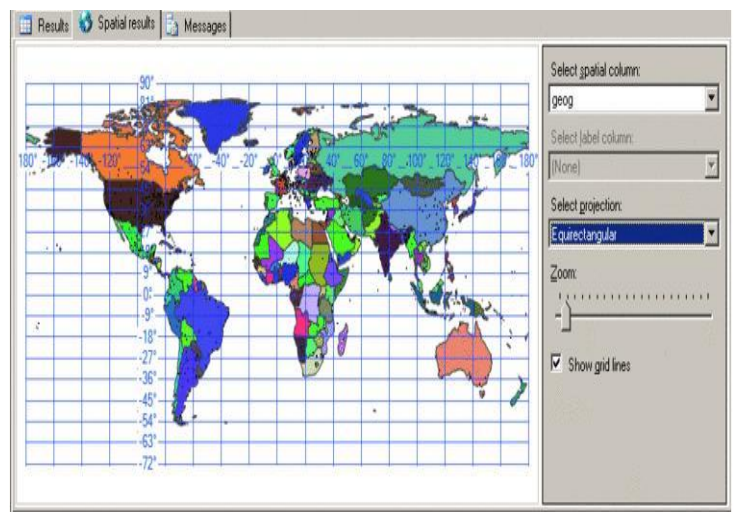

Fig. 6. Output of SQL Server query (B)

\section{A. Application to Common Table Expression (CTE)}

A CTE can be thought of as a temporary result set and are similar to a derived table in that it is not stored as an object and lasts only for the duration of the query. A CTE is generally considered to be more readable than a derived table and does not require the extra effort of declaring a Temp Table while providing the same benefits to the user. However; a CTE is more powerful than a derived table as it can also be selfreferencing, or even referenced multiple times in the same query.

The basic syntax structure for a CTE is shown below:

WITH MYCTE

AS ( SELECT EmpID, FirstName, LastName, ManagerID

FROM Employee

WHERE ManagerID IS NULL)

SELECT *

FROM MYCTE

Building a Recursive CTE

In the following examples, we will show how to harness the power of a recursive CTE query by fulfilling a common business requirement, retrieving hierarchical data. By the time the final query is complete you will be able to easily determine how many levels from the top executive each employee is. A recursive CTE requires four elements in order to work properly.

1. Anchor query (runs once and the results 'seed' the Recursive query)

2. Recursive query (runs multiple times and is the criteria for the remaining results)

3. UNION ALL statement to bind the Anchor and Recursive queries together.

4. INNER JOIN statement to bind the Recursive query to the results of the CTE.

The syntax structure is as follows,

WITH MyCTE

AS ( SELECT EmpID, FirstName, LastName, ManagerID

FROM Employee

WHERE ManagerID IS NULL

UNION ALL

SELECT EmpID, FirstName, LastName, ManagerID

FROM Employee

INNER

JOIN MyCTE ON Employee.ManagerID = MyCTE.EmpID

WHERE Employee.ManagerID IS NOT NULL ) SELECT *

FROM MYCTE.

\section{V.CONCLUDING REMARKS}

The above literature covers the spatial data and tools that are used to tackle the visualization aspect of spatial database. The tools include SQL SERVER 2008. In addition, the different concepts that these tools use are also mentioned. The effective and advanced applications can be developed using the features of SQL SERVER Spatial like ship tracking system and city bus management system.

\section{REFERENCES}

[1] A. Janecek, T. Jordan and F. B. Lima-Neto, "Agent-Based Social Simulation and PSO," Advances in Swarm Intelligence. Springer Berlin Heidelberg, pp. 63-73, 2013.

[2] J. Kennedy, "Particle swarm optimization," In Encyclopedia of Machine Learning, Springer US, pp. 760-766, 2010.

[3] M. G. Epitropakis, P. P.Vassilis and N. V. Michael, "Evolving cognitive and social experience in particle swarm optimization through differential evolution," IEEE Congress on Evolutionary Computation (CEC), 2010.

[4] P. Soni and M. Choudhary, "Hybrid Computational Intelligence for Optimization Based On PSO and DE," International Journal of Advanced Research in Computer Engineering \& Technology, pp-265, 2012.

[5] H. Ahmed and G. Janice, "Swarm Intelligence: Concepts, Models and Applications," Technical Report, 2012-585. Queen's University, Kingston, Ontario, Canada K7L3N6, 2012.

[6] S.R. Dubey and A.S. Jalal, "Robust Approach for Fruit and Vegetable Classification", Procedia Engineering, 38, pp. 3449-3453, 2012.

[7] A. E. Yilmaz, "Swarm Behavior of the Electromagnetics Community as regards Using Swarm Intelligence in their Research Studies," Acta Polytechnica Hungarica, 2010.

[8] S.R. Dubey and A.S. Jalal, "Detection and Classification of Apple Fruit Diseases Using Complete Local Binary Patterns", In Third International Conference on Computer and Communication Technology, pp. 346351, 2012. 
[9] J. P. Gupta, N. Singh, P. Dixit, V. B. Semwal, and S. R. Dubey, "Human Activity Recognition using Gait Pattern," International Journal of Computer Vision and Image Processing, vol. 3, no. 3, pp. 31 $-53,2013$.

[10] M. Sati, V. Vikash, V. Bijalwan, P. Kumari, M. Raj, M. Balodhi, P. Gairola and V.B. Semwal, "A fault-tolerant mobile computing model based on scalable replica", International Journal of Interactive Multimedia and Artificial Intelligence, 2014.

[11] K.K. Susheel, V.B. Semwal and R.C. Tripathi, "Real time face recognition using adaboost improved fast PCA algorithm," arXiv preprint arXiv: 1108.1353 (2011).

[12] K.S. Kumar, V.B. Semwal, S. Prasad and R.C. Tripathi, "Generating 3D Model Using 2D Images of an Object," International Journal of Engineering Science, 2011.

[13] N. Singh, S.R. Dubey, P. Dixit and J.P. Gupta, "Semantic Image Retrieval by Combining Color, Texture and Shape Features," In the Proceedings of the International Conference on Computing Sciences, pp. 116-120, 2012.

[14] V.B. Semwal, V.B. Semwal, M. Sati and S. Verma, "Accurate location estimation of moving object in Wireless Sensor network," International Journal of Interactive Multimedia and Artificial Intelligence, vol. 1, no. 4 , pp. 71-75, 2011.

[15] S.R. Dubey and A.S. Jalal, "Adapted Approach for Fruit Disease Identification using Images," International Journal of Computer Vision and Image Processing, vol. 2, no. 3, pp. 44-58, 2012.

[16] S. Singh and V. Bijalwan, "Design Of Wireless Sensor Network Node On Zigbee For Water Level Detection", Vol. 3, No. 8, 2013.

[17] A. Bijalwan and V. Bijalwan, "Examining the Crimninology using Network Forensic", National Conference USCSTC, 2013.

[18] V. Bijalwan, V. Kumar, P. Kumari and J. Pascual, "KNN based Machine Learning Approach for Text and Document Mining", International Journal of Database Theory and Application, Vol. 7, No. 1, pp. 61-70, 2014.

[19] P. Kumari and V. Pareek, "RAKSHITA- A Novel web based Approach for Protecting Digital Copyrights Using Public Key Digital Watermarking and Human Fingerprints", International conference on methods and models in computer science, 2010.

[20] P. Kumari and A. Vaish, "Instant Face detection and attributes recognition" International Journal of Advanced Computer Science and Applications, 2011.

[21] P. Kumari and A. Vaish, "A Comparative study of Machine Learning algorithms for Emotion State Recognition through Physiological signal", Advances in Intelligent Systems and Computing, Vol. 236, 2013.

[22] P. Kumari and A. Vaish, "Brainwave's Energy feature Extraction using wavelet Transform", proceeding of IEEE SCEECS, 2014

[23] V.B. Semwal, K.S. Kumar, V.S. Bhaskar and M. Sati, "Accurate location estimation of moving object with energy constraint \& adaptive update algorithms to save data", arXiv preprint arXiv:1108.1321, 2011.

[24] J.P. Espada et al., "Virtual Objects on the Internet of Things", International Journal of Interactive Multimedia and Artificial Intelligence, 2011.

[25] R.G. Crespo, S.R. Aguilar, R.F. Escobar and N. Torres, "Dynamic, ecological, accessible and 3D Virtual Worlds-based Libraries using OpenSim and Sloodle along with mobile location and NFC for checking in", International Journal of Interactive Multimedia \& Artificial Intelligence, Vol. 1, No. 7, 2012.

[26] K.S. Kumar et al, "Sports Video Summarization using Priority Curve Algorithm", International Journal on Computer Science \& Engineering, 2010.

[27] R.G. Crespo et al., "Use of ARIMA mathematical analysis to model the implementation of expert system courses by means of free software OpenSim and Sloodle platforms in virtual university campuses", Expert Systems with Applications, Vol. 40, No. 18, pp. 7381-7390, 2013.

[28] S.R. Dubey, P. Dixit, N. Singh, and J.P. Gupta, "Infected fruit part detection using K-means clustering segmentation technique", International Journal of Artificial Intelligence and Interactive Multimedia, Vol. 2, No. 2, 2013.

Vinay S. Bhaskar has been severed as a PCC member in several International conferences and Journals. He is graduated from IIMS pune and serving birla tyre as HR\&PR manager. Previously he served the organization named ITc, Malva Machine Tool etc.

Abhishek Kumar Singh is presently working as Software Engineer, Honeybell, India. He completed his M.Tech form Indian Institute of Information Technology, Allahabad, his major research work Interest in Image Processing, computer sensor network \& Pattern Recognition.

Jyoti Dhruw presently working as Assistant Professor, India. She is B.Tech from CSIT, Durg, Chhattisgrah and pursing M.Tech from NITTR Chandigrah. Her major research work Interest in Image Processing, Sensor Network, Design and Analysis of Algorithm, Data Structure.

Anubha Parashar received her B.Tech in Computer Science and Engineering from ${ }^{4}$ Maharshi Dayanand University, Rohtak. Her research interests include Pattern recognition, Cloud Computing, Big Data and Distributed Database.

Mradula Sharma is currently working as assistant professor at JERC, Jaipur and she completed her M.tech. from MNNIT Allahabad. Her majors are data structure, business intelligency. 